\title{
Improved Evaluation of Planar Calibration Standards Using the TDR Preselection Method
}

\begin{abstract}
J. Vancl
Calibration and correction methods for the Vector Network Analyzer (VNA) are based on the fundamental assumption of the constant error model, which is independent of connected calibration standards and/or devices under test (DUT). Unfortunately, this assumption is not satisfied well for planar calibration standards fabricated by etching technology on soft substrates. An evaluation of the error model is affected especially by variations in the manufacturing process and also by the reproducibility of an assembly. In this paper, we propose error minimization by selecting the best combination of available calibration standards based on time domain reflection (TDR) measurement, which can also be obtained by the fourier transformation from the measured S-parameters. The proposed method was verified experimentally using short, open, load and thru (SOLT) standards fabricated on an FR4 laminate substrate which achieves the essential reduction of the measurement error in the frequency range up to $15 \mathrm{GHz}$.
\end{abstract}

Keywords: Calibration standards, equivalent circuits, error correction, scattering parameters, vector network analyzer.

\section{Introduction}

Many papers on improving VNA calibration procedure have been published recently. The accuracy of the error model is increased by using a CAD-based evaluation of the calibration standards [1] and statistical processing of measured data of an over-determined set of calibration standards [2]-[5]. Another improved calibration procedure optimizes parameterized models of calibration standards to minimize nonreciprocity in a known asymmetrical reciprocal two-port device [6]. These techniques increase the accuracy of the measured S-parameters while maintaining the requirements on the hardware quality of the calibration standards at the same level.

However, the measurement accuracy is substantially worse for standards fabricated on a soft planar substrate than is much longer than a quarter wavelength. Such an effect can be seen clearly in [2], where the $95 \%$ confidence limit of the measured S-parameters is approximately ten times greater for a Rogers 4350B soft substrate than for the measurement carried out using HTCC material and a wafer-probe connection.

The problem of measuring the S-parameters on a planar lossy medium can be overcome by using the TDR preselection method proposed in this paper. Also, an origin of errors is explained assuming the SOLT calibration and correction method.

\section{Error sources}

A typical of S-parameter measurement arrangement is depicted in Fig. 1. An intrinsic calibration standard and a device under test (DUT) are connected to VNA ports through feeding lines and connectors. The intrinsic calibration standard is assumed here as a lumped circuit. The connectors are either soldered to the printed circuit board (PCB) or they just touch the strip when a text fixture is used. The reference planes are usually considered at the center of the PCB.
The overall error of VNA calibration is composed of several particular errors that are described in this section. Each of the particular errors can be assigned to a certain region of the PCB shown in Fig. 1.

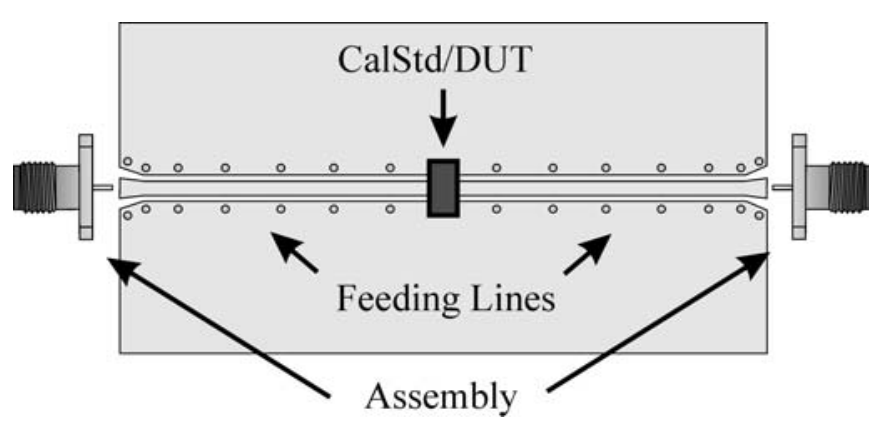

Fig. 1: Important regions of calibration and/or measurement error sources

\subsection{Variation of the manufacturing process}

The limited accuracy of the manufacturing process affects mainly the cross-section of the feeding lines (see Fig. 1). The impedance of the feeding lines differs between items in the calibration kit. Since the feeding lines are naturally distributed circuits, a small impedance difference of 1-2 ohms leads to an error of hundredths in the measured $S$ parameters.

A symptom of this error is the "fast rippled" reflection coefficient of a unitary reflecting standard that is shifted from the reference plane of the calibration standards. Fig. 2 shows an example of a typical tolerance zone for a shifted-short verification standard.

It should be emphasized that it is not possible to correct this error with a different (better) characterization of the calibration standards. 


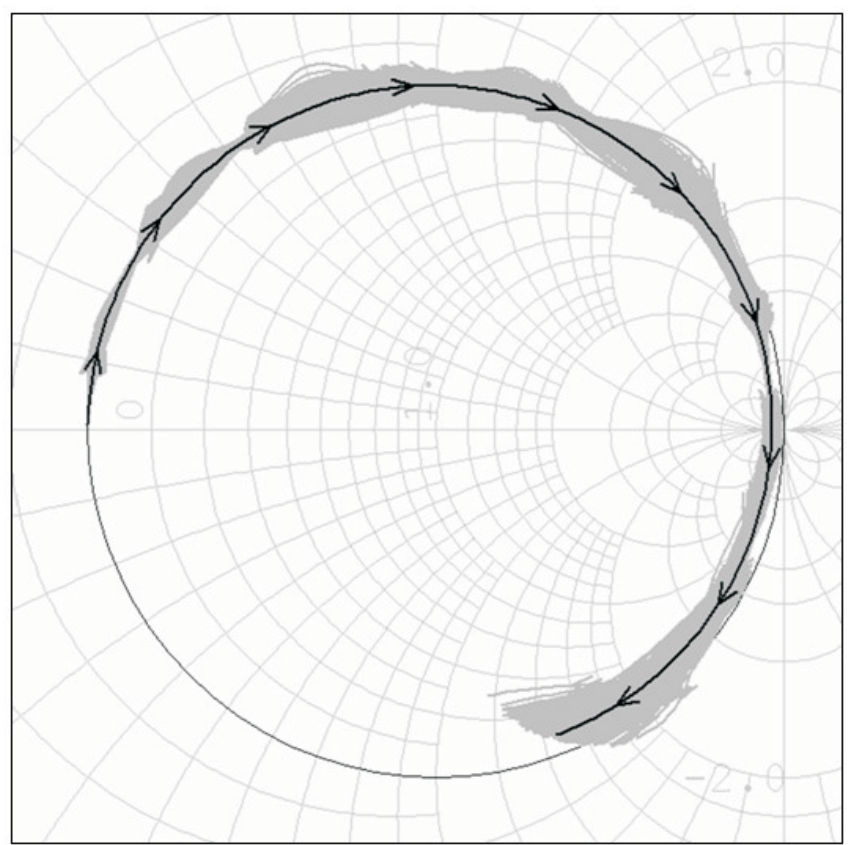

Fig. 2: Typical tolerance zone of the reflection coefficient of the shifted-short verification standard due to variable impedance of the feeding lines

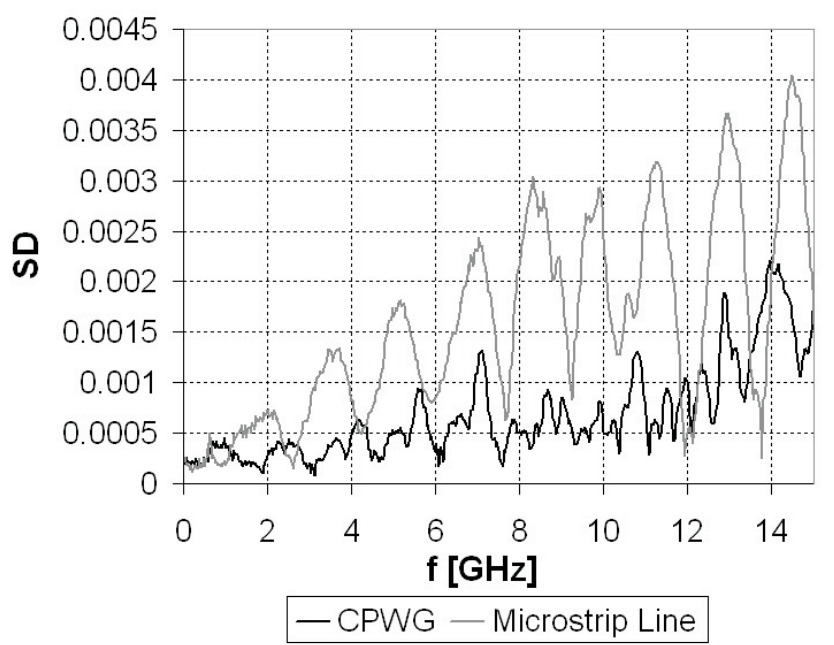

Fig. 3: Typical standard deviations of measured data due to imperfect reproducibility of the assembly (FR4 substrate inserted into the test fixture)

\subsection{Reproducibility of the assembly}

Non-reproducibility of the assembly is minimized by an averaging over several assemblies. An example of standard deviation of measured data for coplanar and microstrip lines is shown in Fig. 3. The contribution to the overall error can be expected at a level of several thousandths.

\subsection{Uncertainty of calibration standards}

A basic set of calibration standards, such as Short, Open and Match, is usually modeled using a full-wave electromagnetic field simulator, taking into account the real geometrical dimensions and the frequency dispersion of the material parameters. Wrong characterization of intrinsic calibration standards leads to spurious "slow ripple" of the measured S-parameters. This error is shown in Fig. 4, where the reflection coefficient of the shifted calibration standards Short2 and Short3 is depicted.

However, uncertainty of intrinsic calibrations standards can be essentially reduced by using an over-determined calibration set, which improves the calibration accuracy by measuring of more distinct calibration standards than the required minimum [5].

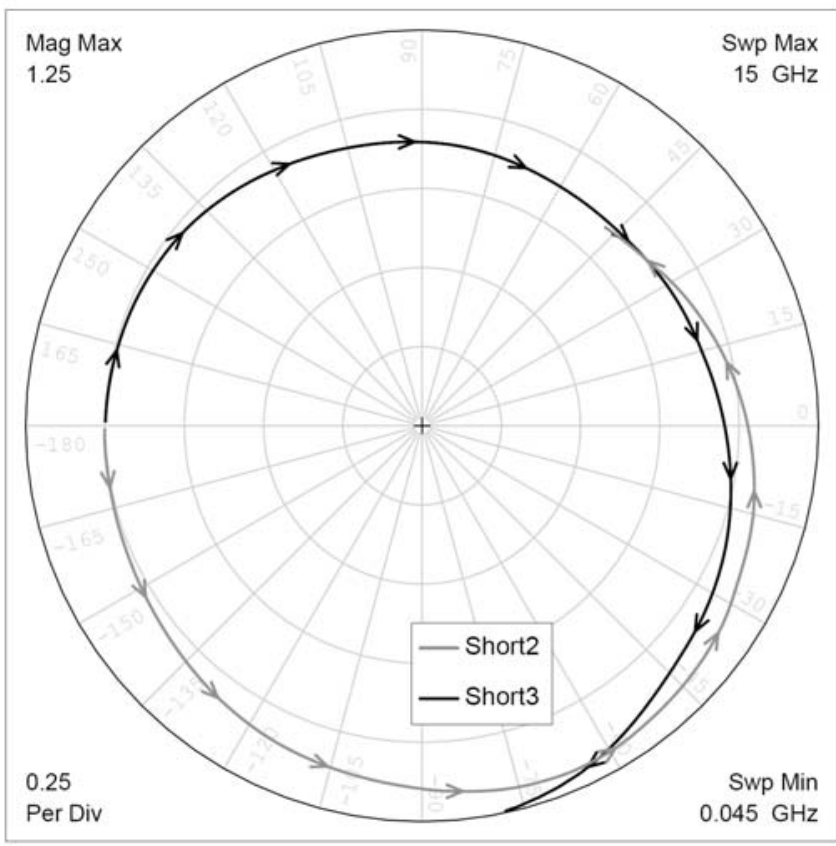

Fig. 4: Reflection coefficient of shifted calibration standards Short2 and Short3

\subsection{Noise}

The smallest error is due to noise generated by VNA. Averaging applied to the measured data can reduce the noise. However, this source of random error is in most cases negligible and thus it is skipped in this paper.

\section{TDR preselection}

Time-domain reflection measurement can provide information about the impedance profile of the transmission line, when the step pulse has a steep enough rise time. Therefore the differences between the impedance profiles of the feeding lines can be obtained. An example of the TDR response of the calibration standards is shown in Fig. 5, where three regions can be distinguished. The response has to be the same in a time window of the cable and feeding line in order to keep the error model constant. Obviously, it is different in the region of intrinsic calibration standards. A zoomed region of the feeding line is shown in Fig. 6.

Usually, several instances of each calibration standard are used for VNA calibration in order to minimize the influence of the variation in the manufacturing process [2]. An alternative approach is to select the best combination of calibration standards with minimum differences of impedance profiles within the feeding-line time window. 


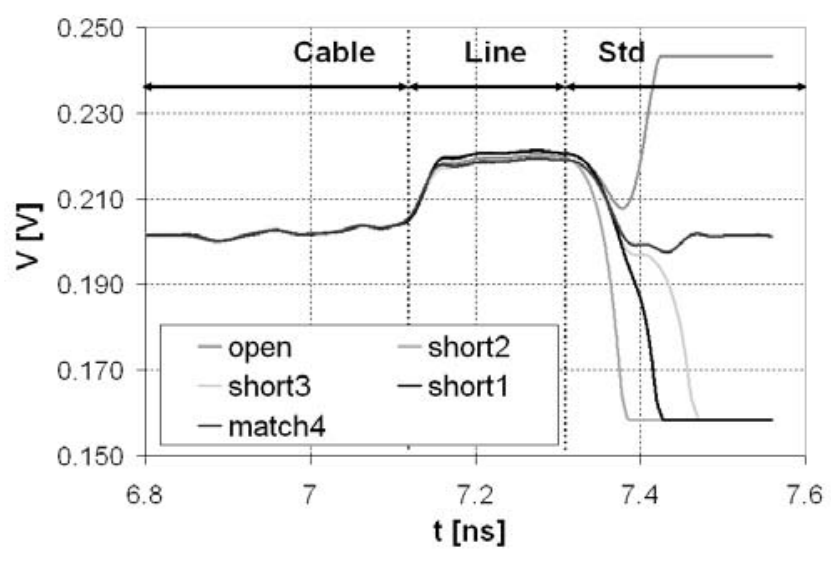

Fig. 5: Time-domain reflection response measurement of calibration standards (FR4 substrate inserted into the test-fixture)

The feeding-line response of the calibration standards can also be obtained by a Fourier transformation of the measured S-parameters. Since we measure S-parameters only up to $15 \mathrm{GHz}$ and the feeding lines are only $25 \mathrm{~mm}$ long, the resolution of the feeding line response is too small for accurate selection of the best combination of calibration standards. However, the differences between the impedance profiles (Fig. 7) are distinguishable and correspond to the TDR measurement (Fig. 6).

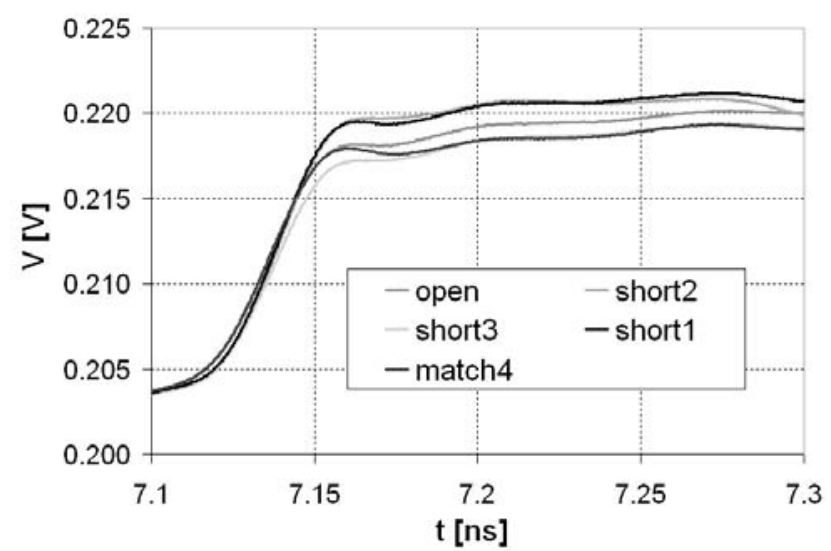

Fig. 6: A detailed time-domain reflection measurement corresponding to the feeding-line response

A suitable criterion for selecting the best combination $C_{B E S T}$ is based on the standard deviation. The criterion formula is

$C_{B E S T}=\arg \min \sum_{j=1}^{N_{T}} \sqrt{\frac{1}{N_{S}-1} \sum_{k=1}^{N_{S}}\left(x_{j, k}-\overline{x_{j}}\right)^{2}}$,

where

$$
\overline{x_{j}}=\frac{1}{N_{S}} \sum_{k=1}^{N_{S}} x_{j, k}
$$

is average value of voltage response in time step $j . N_{S}$ and $N_{T}$ is number of calibration standards and number of time steps, respectively. Total number of possible combinations $n$ is $N_{I}$ raised to the power $N_{S}$, where $N_{I}$ is the number of instances of each calibration standard.

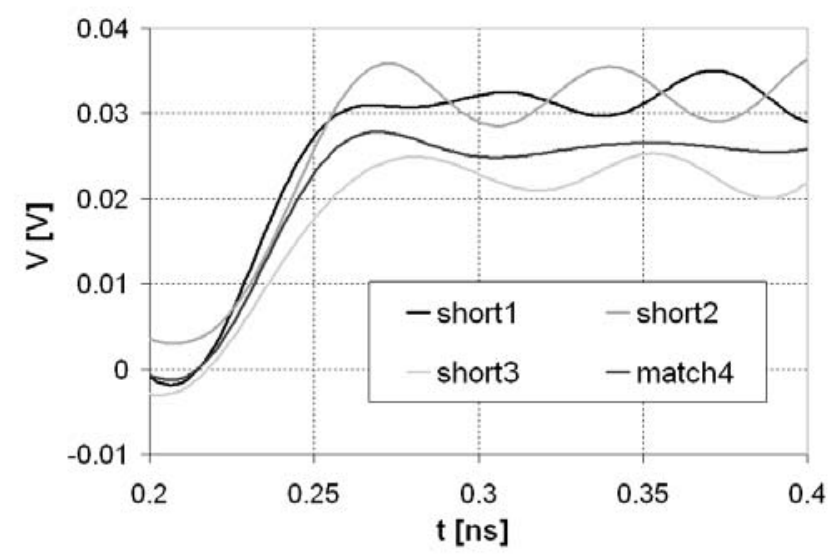

Fig. 7: A detailed feeding-line response obtained by Fourier transformation of the measured S-parameters of the calibration standards

An advantage of the TDR preselection method is that for a given number $N_{I}$ it is possible to achieve the same or smaller variance of the feeding-line impedance profile in comparison with statistical methods. This is because statistical methods take into account all instances, while the TDR preselection method selects the combination with the lowest possible variance.

\section{Calibration scheme}

TRL and SOLT are the most frequently used calibration methods. TRL uses an 8-term error model and therefore it does not enable crosstalk to be corrected. Moreover, the TRL method cannot be used in conjunction with a fixed length test-fixture. On the other hand, calibration of VNA using TRL is much in terms of requirements on manufacturing and evaluation of calibration standards. The SOLT method enables correction of crosstalk, but realization of broadband load is difficult. This problem can be overcome by using the sliding load principle, but it is not suitable for calibration standards manufactured on soft substrates because of poor reproducibility of the reflection coefficient when the load is slid along the transmission line. Another solution is it to combine the two methods, where the broadband fixed load is characterized using the TRL method and DC measurement of the fixed load resistance [7]. Thus the SOLT calibration kit containing the broadband fixed load is the optimal solution for the calibration procedure on soft substrates.

The calibration procedure proposed in this paper is similar to the procedure used in [6], except for the criterion function for the optimization step. The flowchart of the new procedure is depicted in Fig. 8 and is described in the following steps:

1) Short, Open and Load calibration standards are designed using the EM simulator, and their equivalent circuits are extracted, see Fig. 9a)-c). The thru standard is considered as ideal.

2) Full two-port correction coefficients are computed using measured and modeled S-parameters of S, O, L and T standards. The 12-term error model is considered.

3) Measured data of shifted short $\mathrm{S} 2$ and shifted open $\mathrm{O} 2$ are corrected using the correction coefficients computed 
in the previous step. The corrected reflection coefficients are compared with the models of $\mathrm{S} 2$ and $\mathrm{O} 2$ shown in Fig. 9d)-e).

4) The parameters of the equivalent circuits are optimized to minimize the difference between the corrected measured $\mathrm{S}$ parameters and modeled the $\mathrm{S}$ parameters of S2, O2.

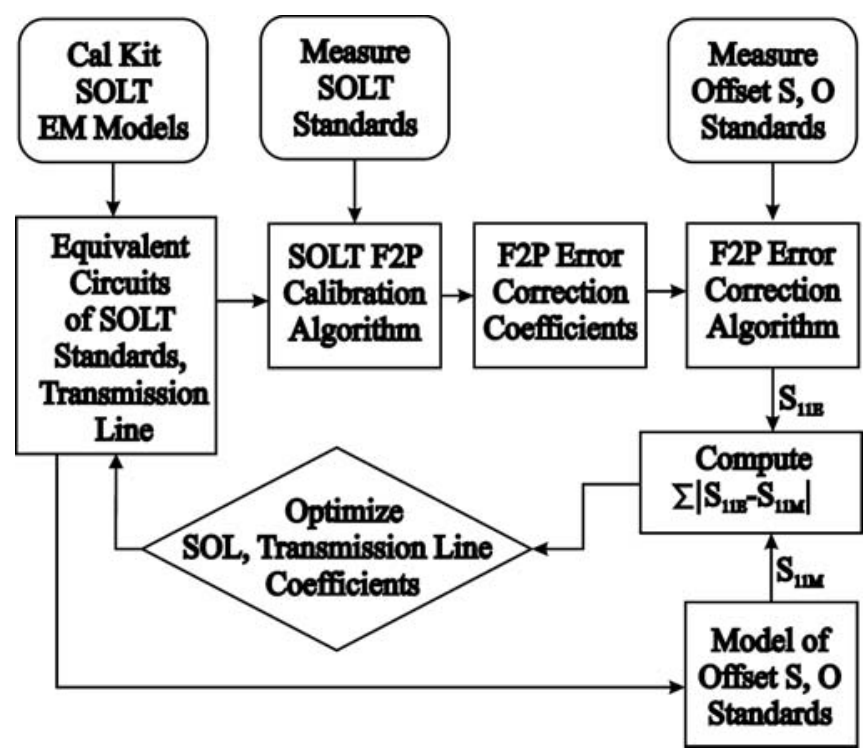

Fig. 8: Flowchart of the process to optimize cal kit parameters.

Involving shifted short/open over determines the characterization of the basic calibration kit, resulting in better accuracy of the $\mathrm{S}$ parameter measurement. The reflection coefficient of a shifted calibration standard, such as S2 and $\mathrm{O} 2$, is much more sensitive than the criterion of reciprocity to improper evaluation of the basic calibration standards $\mathrm{S}, \mathrm{O}$ and $\mathrm{L}$. Thus the difference between estimated and modeled reflection coefficients of $\mathrm{O} 2$ and $\mathrm{S} 2$ was used instead of the reciprocity criterion used in [6].

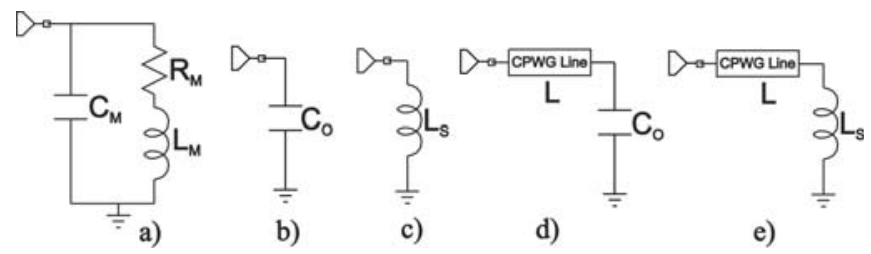

Fig. 9: Equivalent circuits of calibration standards; (a) fixed load, (b) open, (c) short, (d) shifted open, (e) shifted short

\section{Experiment}

An experimental test of the proposed calibration method was carried out using CPWG standards manufactured on an FR4 substrate, each standard in six instances, see Fig. 10.

SHORT 1, OPEN, MATCH 4 and THRU were used as a basic calibration set, SHORT 2 and OPEN 2 were used for optimizing the values of $\mathrm{C}_{\mathrm{M}}, \mathrm{R}_{\mathrm{M}}, \mathrm{L}_{\mathrm{M}}, \mathrm{C}_{\mathrm{O}}$ and $\mathrm{L}_{\mathrm{S}}$ (see Fig. 9), while SHORT 3 was used as a verification standard.

The Agilent $86100 \mathrm{C}$ wideband scope including TDR module 54754A (18 GHz) was used for measuring the TDR
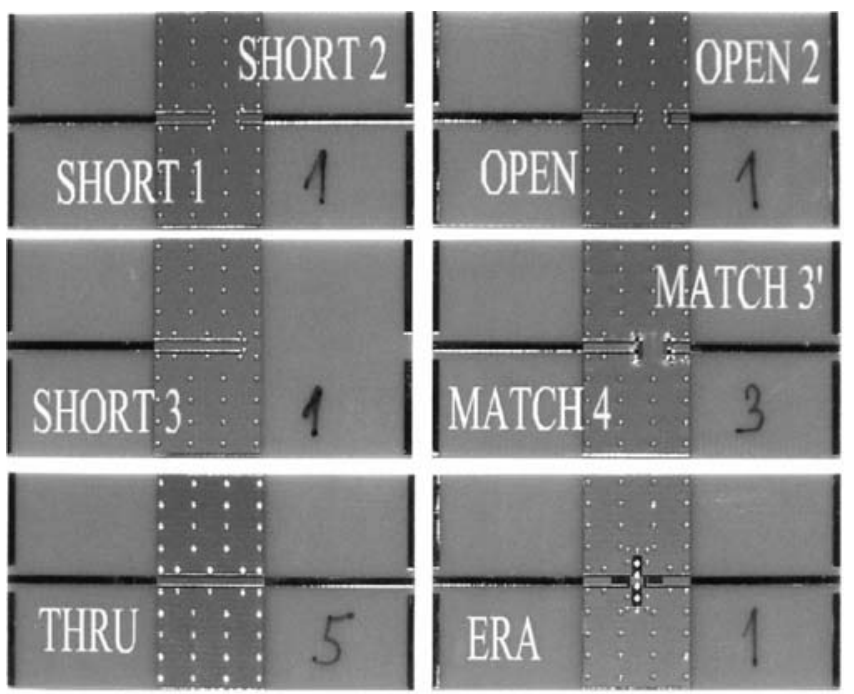

Fig. 10: CPWG calibration standards manufactured on an FR4 substrate $\left(50 \times 25 \times 0.8 \mathrm{~mm}, \varepsilon_{\mathrm{r}}=4.7\right)$

response. The choice of the best combination was carried out in Matlab in according to formula (1). Agilent E8364A PNA was used for measuring the $S$ parameters, and the calibration procedure was carried out according to the flowchart shown in Fig. 8. PCBs of the calibration standards were inserted during measurement into the test fixture attached with SMA connectors. Each calibration standard was measured five times and the data was averaged in order to suppress the influence of the assembly.

The experimental results for the best and worst combination of calibration standards are shown in Fig. 11 and

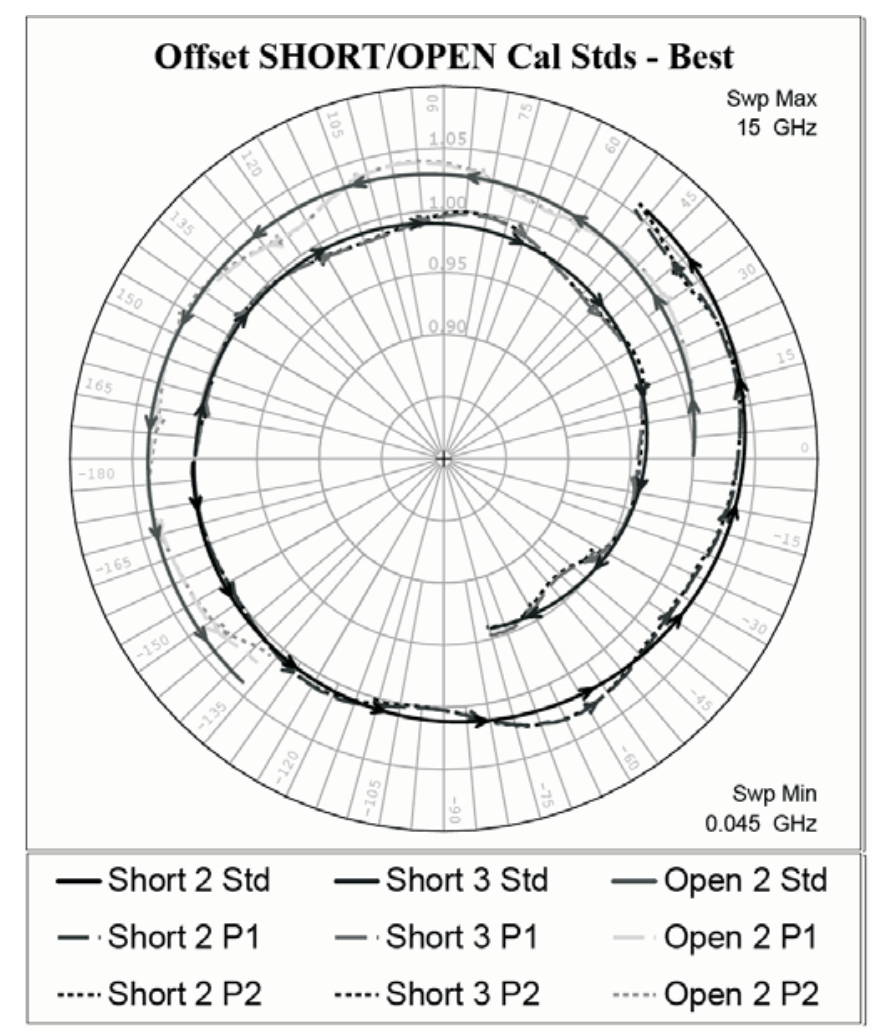

Fig. 11: Reflection coefficient of shifted calibration/verification standards - the best combination 


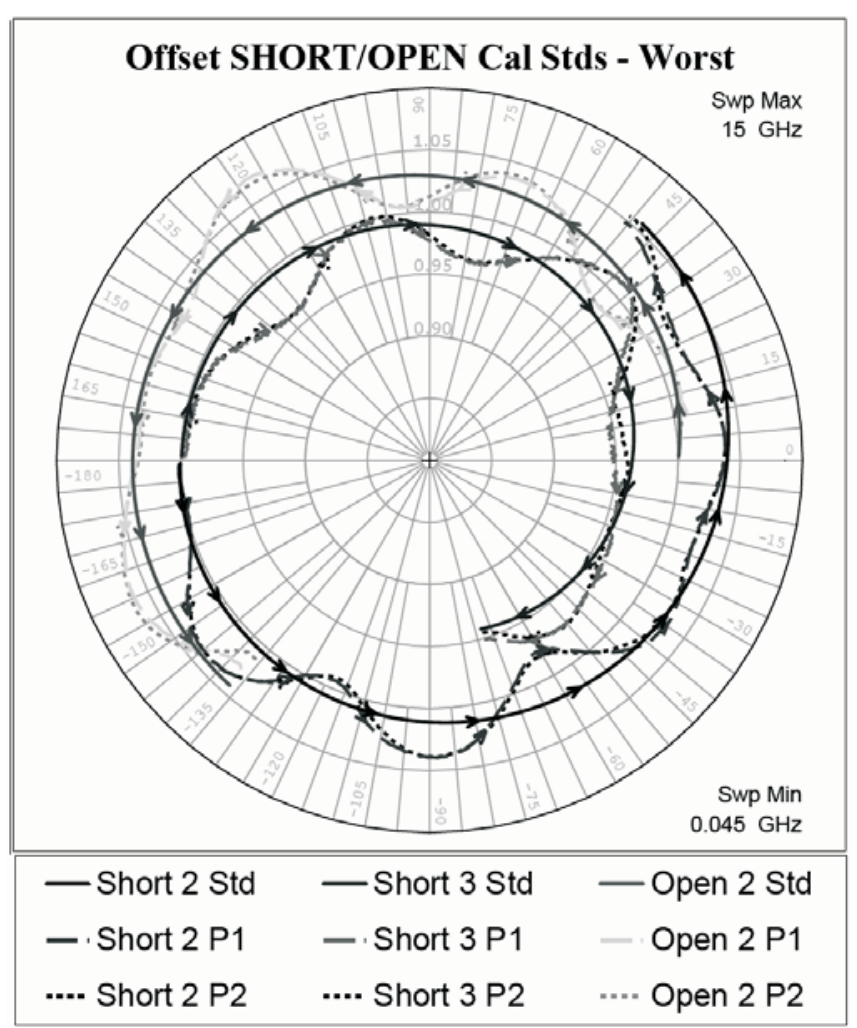

Fig. 12: Reflection coefficient of shifted calibration/verification standards - the worst combination

Fig. 12, respectively. It can be clearly seen that the preselection method essentially reduces the "fast ripple" of the measured reflection coefficients. We estimate that the final uncertainty of measurement is \pm 0.02 in magnitude and $\pm 2^{\circ}$ in angle.

\section{Conclusion}

A improved algorithm for evaluating of calibration standards using the new TDR preselection method was carried out and experimentally verified. The experiment showed that the measurement error of $S$ parameters can be reduced even by a factor of two when the worst and best cases are compared. In contrast to statistical methods, only one set of calibration standards is necessary for recalibring of the VNA.

\section{Acknowledgment}

The research described in this paper was supervised by Ing. V. Sokol, Ph.D., FEE CTU in Prague and supported by research program MSM6840770015 "Research of Methods and Systems for Measurement of Physical Quantities and Measured Data Processing" of CTU in Prague, sponsored by the Ministry of Education and Sports of the Czech Republic, and by grants 102/04/1079 and 13/03014/13117 of the Grant Agency of the Czech Republic.

\section{References}

[1] Deal, W. R., Farkas, D. S.: CAD-based Method to Develop and Evaluate Calibration Standards. IEEE Microwave Magazine, June 2006, p. 70-84.

[2] Chen, X.: Statistical Analysis of Random Errors from Calibration Standards. IEEE MTT-S, Long Beach, CA, June 2005.

[3] Wong, K.: Uncertainty Analysis of the Weighted Least Squares VNA. 64 ${ }^{\text {th }}$ ARFTG Conference, Orlando, 2004.

[4] Stumper, U.: Uncertainty of VNA S-Parameter Measurement Due to Nonideal TRL Calibration Items, IEEE Transaction on Instrumentation and Measurement, Vol. 54, April 2005, p. 676-679.

[5] Satler, M. J., Ridler, N. M., Harris, P. M.: Over-determined Calibration Schemes for RF Network Analysers Employing Generalized Distance Regression, 62 ${ }^{\text {th }}$ ARFTG Conference, Boulder, 2003, p. 127-140.

[6] Scott, J. B.: Investigation of a Method to Improve VNA Calibration in Planar Dispersive Media Through Adding an Asymmetrical Reciprocal Device, IEEE MTT, Vol. 53, September 2005, p. 3007-3013.

[7] Padmanabhan, S., Dunleavy, L., Daniel, J. E., Rodrígues, A., Kirby, P. L.: Broadband Space Conservative On-Wafer Network Analyzer Calibration With More Complex Load and Thru Models. IEEE MTT, Vol. 54, September 2006, p. 3583-3593.

[8] Vancl, J., Sokol, V., Hoffmann, K., Škvor, Z.: Improved Evaluation of Planar Calibration Standard Using TDR Preselection Method, $68^{\text {th }}$ ARFTG Conference, Broomfield, 2006.

Ing. Jan. Vancl

e-mail: vanclj1@fel.cvut.cz

Department of Electromagnetic Field

Czech Technical University in Prague

Faculty of Electrical Engineering

Technická 2

16627 Prague, Czech Republic 